\title{
ACAROLOGY
}

\section{Aranhas e Ácaros Predadores em Copas de Tangerineiras Montenegrina, Mantidas sob Manejo Orgânico, em Montenegro, RS}

\author{
Rosana M. de Morais ${ }^{1}$, Ricardo OtT ${ }^{2}$, Ana P. Ott ${ }^{3}$ e Luiza R. Redaelli ${ }^{1,3}$ \\ ${ }^{1} P P G$ Biologia Animal, Depto. Zoologia, UFRGS, Av. Bento Gonçalves 9500, 91501-970, Porto Alegre, RS \\ entomorais@yahoo.com.br \\ ${ }^{2}$ Museu de Ciências Naturais, Fundação Zoobotânica do Rio Grande do Sul, Rua Dr. Salvador França 1427 \\ 90690-000, Porto Alegre, RS \\ ${ }^{3}$ Depto. Fitossanidade, UFRGS, Av. Bento Gonçalves 7712, 91540-000, Porto Alegre, RS, luredael@ufrgs.br
}

Neotropical Entomology 36(6):939-948 (2007)

Spiders and Predatory Mites in the Canopies of Organically Managed Montenegrina Tangerine Trees, in Montenegro County, RS

\begin{abstract}
A survey was conducted to study the spider and predatory mite fauna occurring in the canopies of tangerine trees (Citrus deliciosa Tenore, cultivar Montenegrina) of an organically managed orchard, at Montenegro County, RS. During a year, fortnightly, 24 randomly trees were selected and sampled in two canopies areas, by using a sheet of white cloth $\left(1 \mathrm{~m}^{2}\right)$. A total of 3,129 arachnids were collected, being 2,559 spiders and 570 mites. Based on the adults, 53 species of Araneae were recognized, belonging to eight families. Among those, the most abundant were Sphecozone sp. (Linyphiidae) (21.8\%) and Chrysso pulcherrima (Mello-Leitão) (Theridiidae) (9.9\%). Autumn exhibited the greatest abundance of young and adults of Araneae (29.2\%) and spring was the season richest in species (40). Margalef and Shannon-Wiener diversity indexes also showed the highest values in spring. The latter index did not show differences among seasons. Simpson complementary index was equal among seasons, reflecting the same degree of species dominance. In Acari, eight species were identified in three families. The most abundant species were the mites Leptus sp.1 (Erythraeidae) (59.4\%) and Amblyseius saopaulus Denmark \& Muma (Phytoseiidae) (30\%). Autumn was the season richest in mite species (eight). The highest abundance $(47 \%)$ was observed on winter and the lowest $(0.88 \%)$ on summer. Among mites, Phytoseiidae showed the highest richness. Among the spiders, Anyphaenidae was the most abundant and Theridiidae had the highest species richness, following the patterns observed for the group.
\end{abstract}

KEY WORDS: Acari, Araneae, diversity, Citrus deliciosa

RESUMO - O estudo objetivou o levantamento da fauna de aranhas e ácaros com hábito predador, em copas de tangerineiras (Citrus deliciosa var. Montenegrina) em pomar orgânico, situado em Montenegro, RS. Durante um ano, quinzenalmente 24 plantas eram sorteadas e avaliadas em duas regiões da copa, utilizando-se um pano-de-batida $\left(1 \mathrm{~m}^{2}\right)$. Capturou-se o total de 3.129 indivíduos, sendo 2.559 aranhas e 570 ácaros. Em Araneae foram reconhecidas 53 espécies adultas distribuídas em oito famílias, dentre estas, as mais abundantes foram Sphecozone sp. (Linyphiidae) $(21,8 \%)$ e Chrysso pulcherrima (Mello-Leitão) (Theridiidae) (9,9\%). No outono observou-se a maior abundância de jovens e adultos $(29,2 \%)$ e, na primavera, a maior riqueza de espécies (40). Na primavera também se registraram os maiores valores para os índices de Margalef e Shannon-Wiener, este último, porém, não diferiu significativamente dos das demais estações. Os valores de Simpson, em todas as estações foram semelhantes, indicando o mesmo grau de dominância das espécies. Em Acari registraram-se oito espécies, distribuídas em três famílias, as mais abundantes foram Leptus sp.1 (Erythraeidae) (59,4\%) e Amblyseius saopaulus Denmark \& Muma (Phytoseiidae) (30\%). O outono foi a estação mais rica em número de espécies (oito). No inverno observou-se a maior abundância de indivíduos (47\%) e, no verão, a menor $(0,88 \%)$. Entre os ácaros, Phytoseiidae apresentou a maior riqueza. Entre as aranhas, Anyphaenidae foi a que apresentou a maior abundância e Theridiidae, a maior riqueza de espécies, seguindo assim o padrão já observado para o grupo.

PALAVRAS-CHAVE: Acari, Araneae, diversidade, Citrus deliciosa 
As aranhas constituem um dos maiores grupos de invertebrados predadores e representam um fator limitante no potencial de crescimento exponencial de populações de insetos (Riechert \& Lockley 1984). Em agroecossistemas, assim como em sistemas naturais, compreendem um conspícuo componente da fauna, sucesso possivelmente relacionado, além do caráter generalista, à alta diversidade de estratégias de captura, as quais são características de cada espécie. Esses atributos fazem com que as aranhas ocupem diferentes nichos e mantenham um forrageamento constante, contribuindo para a redução de vários grupos de presas (Green 1996).

Hajaj et al. (2000) estudando comunidades de artrópodes arborícolas em ambiente natural, constataram que as aranhas foram fortemente limitadas pela forma e qualidade de seu hábitat, tanto que a riqueza de espécies e o tamanho corporal tendem a serem maiores quando a complexidade do meio é elevada, resultando em distintos padrões de ocupação entre as guildas. Pomares de citros, mesmo oferecendo pouca diversidade de espécies vegetais, comparativamente a um sistema natural, têm um caráter perene proporcionando micro-hábitats estáveis. Além disso, o porte das plantas é grande o suficiente para abrigar populações de aranhas, que, assim, não necessitam emigrar para dispersarem-se (Green 1996).

Em estudos realizados nos Estados Unidos da América (EUA) (Amalin et al. 2001), foi observado o comportamento alimentar de Hibana velox (Becker) (Anyphaenidae), Cheirachantium inclusum (Henz) (Miturgidae) e Trachelas volutus Gertsch (Corinnidae), como predadoras de Phyllocnistis citrella Stainton (Lepidoptera: Gracillariidae), espécie que, em sua fase imatura, causa significativos danos à citricultura (Gravena 1994). A capacidade que essas aranhas demonstraram de encontrar a larva abaixo da epiderme foliar reforça a possibilidade do seu emprego na redução da população do lepidóptero.

A acarofauna é comumente estudada em sistemas agrícolas com relação às espécies fitófagas e transmissoras de doenças (Flechtmann 1989), apesar da ação de ácaros como inimigos naturais de pragas já ser conhecida há muito tempo (Moraes 2002). Ácaros predadores são freqüentemente encontrados associados a ácaros fitófagos em culturas anuais e perenes, assim como em vegetações nativas (Ferla \& Moraes 2002). Em pomares de citros, os predadores são extremamente importantes para a manutenção do equilíbrio das populações de cochonilhas e principalmente de outros ácaros, como o ácaro-da-falsa-ferrugem (Phyllocoptruta oleivora Ashmead, Eriophyidae) e o ácaro-da-leprose (Brevipalpus phoenicis Geijskes, Tenuipalpidae) (Parra et al. 2003), sendo este último considerado praga-chave na cultura (Raga et al. 1996).

Várias são as famílias de Acari que abrigam espécies predadoras, no entanto Phytoseiidae tem recebido considerável atenção pelo potencial como agente de controle biológico de espécies fitófagas em diversas plantas cultivadas (McMurtry \& Croft 1997), talvez pelo fato de suas espécies serem abundantes (Parra et al. 2003) ou capazes de realizar movimentos rápidos e busca ativa pelas presas (Moraes 2002).
Em regiões tropicais e subtropicais, há um elevado número de espécies e conseqüentemente um grande complexo de predadores (Green 1996), que potencialmente podem ser usados no manejo e controle de espécies-praga. Este aspecto tem relevância especialmente, na cultura dos citros a qual é de elevada importância para a economia do país, por ser o Brasil um dos principais produtores citrícolas (IBGE 2005). O presente estudo visou ampliar o conhecimento acerca dos aracnídeos associados à cultura de citros no sul do país, avaliando a composição, abundância e riqueza de espécies presentes em um pomar orgânico em Montenegro, RS.

\section{Material e Métodos}

O estudo foi desenvolvido em um pomar de tangerineira da variedade Montenegrina (Citrus deliciosa Tenore) $\left(29^{\circ} 37^{\prime} 51^{\prime \prime} \mathrm{S}\right.$ e $\left.51^{\circ} 28^{\prime} 10^{\prime \prime} \mathrm{W}\right)$, situado no município de Montenegro, pertencente à região da Depressão Central, cuja topografia é levemente ondulada, com menos de $100 \mathrm{~m}$ de altitude. O clima da região é, segundo a classificação de Köppen, do tipo Cfa subtropical úmido, com precipitações bem distribuídas no ano, sofrendo a influência de massas de ar tropicais e polar-atlânticas. A temperatura média anual é de $19,1^{\circ} \mathrm{C}$, com a máxima média de $25,8^{\circ} \mathrm{C}$, mínima média de $14,4^{\circ} \mathrm{C}$ e a precipitação média é de $1.424 \mathrm{~mm} /$ ano (IPAGRO 1989).

O pomar possui 14 anos de idade, uma área de 0,6 ha e aproximadamente 300 plantas, distribuídas no espaçamento de três metros entre as plantas e seis entre fileiras. Desde sua instalação é mantido sob manejo orgânico, recebendo aplicações de chorume e composto orgânico, ambos provenientes da Usina de Compostagem da Cooperativa dos Citricultores Ecológicos do Vale do Caí (ECOCITRUS), além de calda bordalesa três vezes por ano e uma aplicação anual de calda sulfocálcica. A vegetação espontânea constituía-se de plantas herbáceas e rasteiras, pertencentes principalmente a Asteraceae, Fabaceae, Poaceae e Solanaceae, as quais eram mantidas nas entrelinhas e entre as árvores, e controladas apenas através de uma roçada anual.

As amostragens foram realizadas quinzenalmente, no período entre 29 de março de 2004 e 14 de março de 2005, totalizando seis ocasiões amostrais no outono/2004, sete no inverno/2004, seis na primavera/2004 e sete no verão/2005. Em cada ocasião eram retiradas, de 24 plantas, previamente sorteadas, duas unidades amostrais (uma em cada face da planta voltada para as entrelinhas). Para coleta dos aracnídeos utilizou-se um pano branco medindo $1 \mathrm{~m}^{2}$, disposto sob a copa da planta; com auxílio de um bastão de madeira, efetuaram-se dez batidas sobre os ramos da copa. O pano era enrolado imediatamente e o seu conteúdo repassado para dentro de um saco plástico etiquetado, constituindo uma unidade amostral. Aranhas adultas foram identificadas em unidades morfológicas afins, constituíndo morfoespécies e, quando possível, identificadas até o nível específico; imaturos foram apenas contabilizados em nível de família. As famílias de aranhas foram classificadas, conforme sua estratégia de caça, em caçadoras errantes e construtoras de teia, segundo Uetz et al. (1999). 
A partir das espécies de aranhas e ácaros, obtidos em cada uma das ocasiões de amostragem, foi construída uma curva de suficiência amostral para cada um dos grupos, utilizando-se o programa estatístico EstimateS versão 7.5 (Colwell 2005). Por meio deste programa, com uma randomização de 500 vezes, também foram obtidos os valores estimados para o esforço amostral, sendo utilizados os estimadores: Bootstrap, que se baseia na proporção de ocasiões amostrais em que cada espécie esteve presente, e Chao 2, que toma como referência o número de espécies presentes em uma ou duas ocasiões durante todo o período amostral (Moreno 2001).

As estações do ano foram comparadas quanto à abundância e riqueza de espécies através de curvas de rarefação, utilizando-se o programa BioDiversity Professional versão 2 (McAleece 1997) e Past versão 1.34 (Hammer et al. 2001). Esta avaliação foi feita apenas com relação aos indivíduos adultos de Araneae, já que o grupo dos ácaros foi praticamente ausente em uma das estações, não permitindo deste modo, comparação com as demais.

\section{Resultados e Discussão}

Ao longo das 26 ocasiões amostrais foram coletados 3.129 aracnídeos (2.559 aranhas e 570 ácaros). Do total de aranhas coletadas, 353 eram indivíduos adultos, a partir dos quais, foram reconhecidas 53 morfoespécies de oito famílias; adicionalmente mais sete famílias foram identificadas exclusivamente com base nos indivíduos jovens (Tabelas $1 \mathrm{e}$ 2). A curva de suficiência amostral para aranhas (Fig. 1) foi ascendente na maior parte do período, sugerindo que um maior número de espécies poderia ainda ser registrado. $\mathrm{O}$ estimador Bootstrap apontou que $87,3 \%$ das espécies esperadas foram amostradas, enquanto Chao 2 as estimou em 78,6\%.

$\mathrm{Na}$ ordem Acari, os 570 indivíduos predadores coletados estiveram distribuídos em três famílias e oito espécies. A curva de suficiência (Fig. 2) evidencia redução no número de espécies capturadas a partir do final do inverno, sugerindo que a continuidade das coletas dificilmente resultaria em novas espécies registradas. Esse fato também foi verificado pelos estimadores, onde Bootstrap indica $89,3 \%$ e Chao 2 que $96,1 \%$ das espécies estimadas para a captura foram coletadas.

O baixo número de ácaros em relação ao de aranhas pode estar relacionado ao método de coleta utilizado, já que em levantamentos de acarofauna são usualmente adotadas outras técnicas específicas para o grupo, com análise mais minuciosa de ramos e folhas (Sato et al. 1994, Ferla \& Moraes 2002).

Erythraeidae $(68,9 \%)$ destaca-se pela abundância de indivíduos em relação às demais famílias de Acari, representada com mais de $80 \%$ por apenas uma espécie. Espécies dessa família são comuns em cobertura herbácea de solo e estão entre os grupos predominantes de artrópodes predadores (Smith et al. 1998). A presença de indivíduos

Tabela 1. Número de aranhas jovens (J) e adultas (A) por estação, número total de indivíduos amostrados em copas de C. deliciosa var. Montenegrina, cultivado sob manejo orgânico, (29³7'51'"S e 51²8'10"W), Montenegro, RS, de março de 2004 a março de 2005, e respectiva estratégia de caça (C, caçadora errante; T, construtora de teia).

\begin{tabular}{|c|c|c|c|c|c|c|c|c|c|c|c|}
\hline \multirow{2}{*}{ Famílias } & \multicolumn{2}{|c|}{ Outono } & \multicolumn{2}{|c|}{ Inverno } & \multicolumn{2}{|c|}{ Primavera } & \multicolumn{2}{|c|}{ Verão } & \multicolumn{2}{|c|}{ Total } & \multirow{2}{*}{ Estratégia de caça } \\
\hline & $\mathrm{J}$ & $\mathrm{A}$ & $\mathrm{J}$ & A & $\mathrm{J}$ & A & $\mathrm{J}$ & A & $\mathrm{J}$ & $\mathrm{A}$ & \\
\hline Anyphaenidae & 375 & 2 & 294 & 6 & 179 & 5 & 171 & 3 & 1019 & 16 & $\mathrm{C}$ \\
\hline Theridiidae & 99 & 25 & 119 & 69 & 127 & 35 & 93 & 23 & 438 & 152 & $\mathrm{~T}$ \\
\hline Salticidae & 102 & 9 & 64 & 15 & 54 & 15 & 131 & 20 & 351 & 59 & $\mathrm{C}$ \\
\hline Araneidae & 52 & 4 & 82 & 3 & 62 & 18 & 57 & 2 & 253 & 27 & $\mathrm{~T}$ \\
\hline Linyphiidae & 11 & 13 & 10 & 25 & 3 & 40 & 7 & 10 & 31 & 88 & $\mathrm{~T}$ \\
\hline Thomisidae & 9 & 1 & 7 & 0 & 6 & 4 & 13 & 2 & 35 & 7 & $\mathrm{C}$ \\
\hline Miturgidae & 27 & 2 & 1 & 0 & 1 & 0 & 1 & 1 & 30 & 3 & $\mathrm{C}$ \\
\hline Oxyopidae & 8 & 0 & 2 & 1 & 4 & 0 & 2 & 0 & 16 & 1 & $\mathrm{C}$ \\
\hline Sparassidae & 4 & 0 & 1 & 0 & 2 & 0 & 3 & 0 & 10 & 0 & $\mathrm{C}$ \\
\hline Psauridae & 2 & 0 & 2 & 0 & 3 & 0 & 0 & 0 & 7 & 0 & $\mathrm{~T}$ \\
\hline Tethragnathidae & 1 & 0 & 4 & 0 & 1 & 0 & 0 & 0 & 6 & 0 & $\mathrm{~T}$ \\
\hline Corinnidae & 0 & 0 & 1 & 0 & 2 & 0 & 2 & 0 & 5 & 0 & $\mathrm{C}$ \\
\hline Mimetidae & 0 & 0 & 0 & 0 & 1 & 0 & 1 & 0 & 2 & 0 & $\mathrm{C}$ \\
\hline Theridiossomatidae & 2 & 0 & 0 & 0 & 0 & 0 & 0 & 0 & 2 & 0 & $\mathrm{~T}$ \\
\hline Lycosidae & 1 & 0 & 0 & 0 & 0 & 0 & 0 & 0 & 1 & 0 & $\mathrm{C}$ \\
\hline Total & 693 & 55 & 587 & 119 & 445 & 118 & 481 & 61 & 2206 & 353 & \\
\hline Total/estação & \multicolumn{2}{|c|}{748} & \multicolumn{2}{|c|}{706} & \multicolumn{2}{|c|}{563} & & & \multicolumn{2}{|c|}{2559} & \\
\hline
\end{tabular}


Tabela 2. Abundância sazonal e total $(\mathrm{N})$, e riqueza $(\mathrm{S})$ de aranhas adultas e ácaros predadores em copas de $C$. deliciosa var. Montenegrina, cultivado sob manejo orgânico, (29³7'51'S e 51²8'10”W), Montenegro, RS, março de 2004 a março de 2005.

\begin{tabular}{|c|c|c|c|c|c|}
\hline \multirow{2}{*}{ Espécies } & \multicolumn{4}{|c|}{ Estações } & \multirow{2}{*}{$\mathrm{N}$} \\
\hline & Outono & Inverno & Primavera & Verão & \\
\hline Araneae & & & & & 353 \\
\hline Anyphaenidae & & & & & 16 \\
\hline Arochosia honesta Keyserling & 1 & 0 & 2 & 1 & 4 \\
\hline Arochosia sp. & 0 & 1 & 2 & 1 & 4 \\
\hline Jessica osoriana (Mello-Leitão) & 1 & 4 & 1 & 1 & 7 \\
\hline Aysha sp. & 0 & 1 & 0 & 0 & 1 \\
\hline Araneidae & & & & & 26 \\
\hline Acacesia hamata (Hentz) & 0 & 0 & 1 & 0 & 1 \\
\hline Alpaida albocincta (Mello-Leitão) & 0 & 0 & 2 & 0 & 2 \\
\hline Alpaida gallardoi Levi & 0 & 0 & 0 & 1 & 1 \\
\hline Alpaida leucograma (White) & 0 & 0 & 1 & 0 & 1 \\
\hline Alpaida rubellula (Keyserling) & 0 & 0 & 1 & 0 & 1 \\
\hline Alpaida veniliae (Keyserling) & 0 & 0 & 1 & 0 & 1 \\
\hline Alpaida sp. & 1 & 1 & 1 & 1 & 4 \\
\hline Cyclosa sp. & 0 & 0 & 1 & 0 & 1 \\
\hline Bertrana rufostriata Simon & 0 & 1 & 7 & 0 & 8 \\
\hline Gasteracantha cancriformis (L.) & 2 & 0 & 0 & 0 & 2 \\
\hline Metazygia aff. viriosa & 0 & 1 & 0 & 0 & 1 \\
\hline Parawixia sp. & 0 & 0 & 3 & 0 & 3 \\
\hline Linyphiidae & & & & & 88 \\
\hline Sphecozone sp. & 13 & 21 & 33 & 10 & 77 \\
\hline Scolecura sp. & 0 & 3 & 2 & 0 & 5 \\
\hline Erigoninae indet. & 0 & 0 & 3 & 0 & 3 \\
\hline Linyphiidae indet. & 0 & 1 & 2 & 0 & 3 \\
\hline Miturgidae & & & & & 3 \\
\hline Cheirachantium inclusum (Hentz) & 2 & 0 & 0 & 1 & 3 \\
\hline Oxyopidae & & & & & 1 \\
\hline Oxyopes salticus & 0 & 1 & 0 & 0 & 1 \\
\hline Salticidae & & & & & 59 \\
\hline Ashtabula sp. & 0 & 1 & 1 & 1 & 3 \\
\hline Beata sp. & 0 & 0 & 1 & 0 & 1 \\
\hline Chira thysbe Simon & 0 & 3 & 1 & 8 & 12 \\
\hline Chira sp. & 1 & 1 & 6 & 2 & 10 \\
\hline Coryphasia sp. & 0 & 2 & 0 & 0 & 2 \\
\hline Cotinusa sp. 1 & 7 & 3 & 3 & 4 & 17 \\
\hline Cotinusa sp. 2 & 1 & 5 & 2 & 5 & 13 \\
\hline Neonella sp. & 0 & 0 & 1 & 0 & 1 \\
\hline
\end{tabular}


Tabela 2. Continuação.

\begin{tabular}{|c|c|c|c|c|c|}
\hline \multirow{2}{*}{ Espécies } & \multicolumn{4}{|c|}{ Estações } & \multirow{2}{*}{$\mathrm{N}$} \\
\hline & Outono & Inverno & Primavera & Verão & \\
\hline Theridiidae & & & & & 152 \\
\hline Achaearanea bellula (Keyserling) & 2 & 1 & 0 & 0 & 3 \\
\hline Achaearanea hirta (Taczanowski) & 4 & 13 & 3 & 5 & 25 \\
\hline Achaearanea passiva (Keyserling) & 0 & 0 & 1 & 0 & 1 \\
\hline Achaearanea tesselata (Keyserling) & 1 & 0 & 0 & 1 & 2 \\
\hline Anelosimus ethicus (Keyserling) & 1 & 2 & 0 & 0 & 3 \\
\hline Anelosimus nigrescens (Keyserling) & 3 & 12 & 2 & 7 & 24 \\
\hline Argyrodes argentatus O. P. Cambridge & 0 & 0 & 0 & 1 & 1 \\
\hline Chrysso pulcherrima (Mello-Leitão) & 8 & 16 & 9 & 2 & 35 \\
\hline Dipoena pumicata (Keyserling) & 0 & 0 & 1 & 0 & 1 \\
\hline Dipoena alta Keyserling & 1 & 9 & 5 & 0 & 15 \\
\hline Episinus sp. 1 & 1 & 0 & 1 & 0 & 2 \\
\hline Episinus sp. 2 & 0 & 2 & 5 & 0 & 7 \\
\hline Emertonella taczanowskii (Keyserling) & 1 & 0 & 2 & 1 & 4 \\
\hline Romphea sp. & 1 & 6 & 1 & 2 & 10 \\
\hline Theridion pernambucum Levi & 0 & 0 & 0 & 1 & 1 \\
\hline Theridion plaumanni Levi & 0 & 3 & 1 & 1 & 5 \\
\hline Theridion positivum Chamberlin & 2 & 3 & 0 & 2 & 7 \\
\hline Theridion sp. & 0 & 0 & 2 & 0 & 2 \\
\hline Wamba crispulus Simon & 0 & 1 & 1 & 0 & 2 \\
\hline Theridiidae sp. & 0 & 1 & 1 & 0 & 2 \\
\hline Thomisidae & & & & & 8 \\
\hline Tmarus sp. & 1 & 0 & 2 & 2 & 5 \\
\hline Misumenops pallens (Keyserling) & 0 & 0 & 2 & 0 & 2 \\
\hline Misumenops sp. & 0 & 0 & 1 & 0 & 1 \\
\hline Acari & & & & & 570 \\
\hline Erythraeidae & & & & & 393 \\
\hline Leptus sp. 1 & 79 & 189 & 71 & 0 & 339 \\
\hline Leptus sp. 2 & 20 & 4 & 11 & 1 & 36 \\
\hline Leptus sp. 3 & 9 & 7 & 1 & 1 & 18 \\
\hline Phytoseiidae & & & & & 176 \\
\hline Amblyseius operculatus DeLeon & 0 & 2 & 0 & 0 & 2 \\
\hline Amblyseius saopaulus Denmark \& Muma & 13 & 62 & 93 & 3 & 171 \\
\hline Iphyseiodes zuluagai Denmark \& Muma & 0 & 1 & 0 & 0 & 1 \\
\hline Typhlodromips mangleae DeLeon & 0 & 2 & 0 & 0 & 2 \\
\hline Cunaxidae & & & & & 1 \\
\hline Cunaxa sp. & 0 & 1 & 0 & 0 & 1 \\
\hline $\mathrm{N}$ & 176 & 387 & 294 & 66 & 923 \\
\hline S & 25 & 36 & 44 & 26 & 61 \\
\hline
\end{tabular}




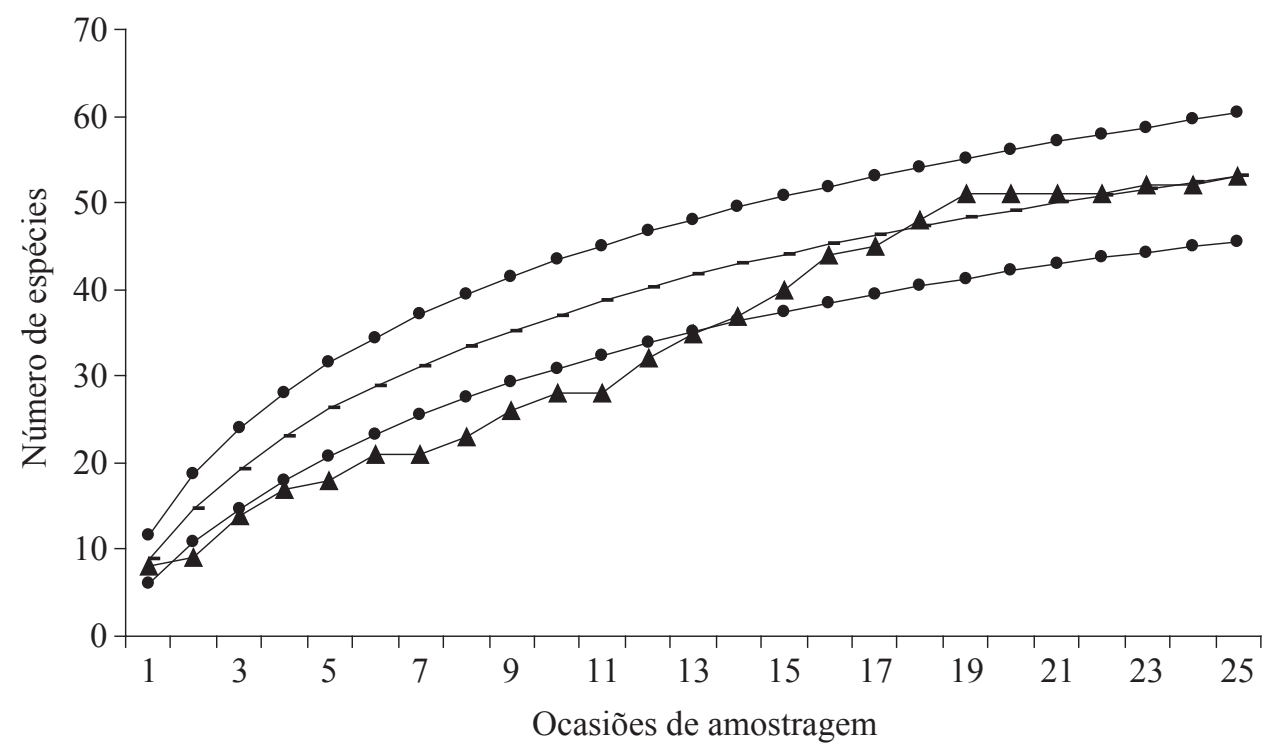

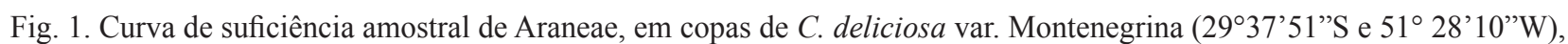
pomar orgânico em Montenegro, RS, de março de 2004 a março de 2005. (--•-- intervalo de confiança; - $\mathbf{\Delta}$ - número acumulado de espécies observado; - número acumulado de espécies randomizado 500 vezes).

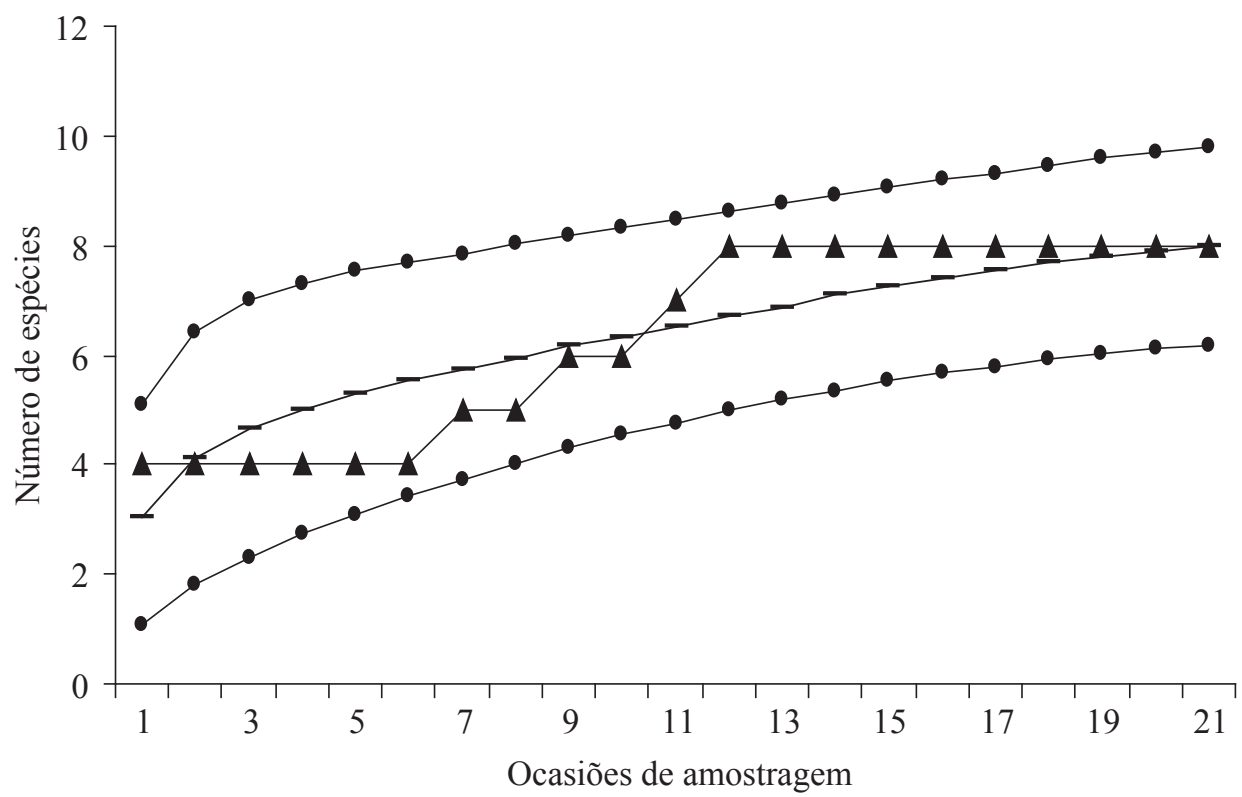

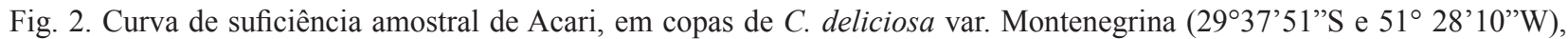
pomar orgânico em Montenegro, RS, de março de 2004 a março de 2005. (--•-- intervalo de confiança; - $\mathbf{\Delta}$ - número acumulado de espécies observado; - número acumulado de espécies randomizado 500 vezes).

dessa família em elevada abundância no presente estudo pode ter decorrido do procedimento de amostragem, no qual o do pano-de-batida permanecia sobre a vegetação herbácea, permitindo a entrada desses organismos (Gilberto Moraes, com. pess.). Apesar de as espécies de Erythraeidae serem estudadas principalmente pelo hábito parasita apresentado na fase imatura (Baker \& Selden 1997), a capacidade de predação dos adultos da família é conhecida. Experimentos em laboratório evidenciaram o elevado índice de consumo por parte de Balaustium spp. (Erythraeidae), quando expostas a ovos de moscas vajereiras (Merfield et al. 2004).

Quanto à riqueza de ácaros, Phytoseiidae representou metade das oito espécies encontradas, seguida por Erythraeidae com três espécies, e Cunaxidae com uma espécie representada por um único indivíduo. Dentre os fitoseídeos, Amblyseius Berlese, foi o gênero mais abundante, já tendo sido registrado em outros cultivos, como macieira (Ferla \& Moraes 1998), goiabeira, ipê e amoreira (Ferla \& Moraes 
2002). Elevada riqueza de Phytoseiidae também foi observada em pomares de macieira no Rio Grande do Sul, representando $77 \%$ das espécies de ácaros predadores registradas (Ferla \& Moraes 1998). Considerando ácaros fitófagos e predadores, Daud \& Feres (2005) constataram que 16\% das espécies amostradas em Mabea fistulifera Mart. (Euphorbiaceae), em dois fragmentos de mata estacional semidecídua em São José do Rio Preto, SP, eram de Phytoseiidae. Da mesma forma, em aceroleiras em Recife, PE, Barbosa et al. (2003) registraram que os fitoseídeos constituíram $6,4 \%$ dos ácaros amostrados. Segundo esses autores os representantes dessa família estariam, possivelmente, contribuindo para a redução de ácaros fitófagos pertencentes a Eriophyidae e Tenuipalpidae. Conforme Moraes (1992) e Parra et al. (2003), é freqüente a presença de diversas espécies fitófagas de eriofiídeos e tenuipalpídeos em pomares de citros. Assim, é possível que indivíduos dessas mesmas famílias estejam contribuindo para a riqueza de fitoseídeos constatada no presente estudo.

Entre as aranhas, as quatro famílias mais abundantes foram: Anyphaenidae (40,4\%), Theridiidae (23\%), Salticidae (16\%) e Araneidae (10,9\%), estando incluídas em diferentes guildas quanto à estratégia de caça que adotam (Young \& Edwards 1990). Anyphaenidae e Salticidae possuem hábito caçador errante, enquanto Theridiidae e Araneidae constroem teias para a captura de presas (Uetz et al. 1999). Essa diferenciação no modo de captura, característica de cada grupo funcional, permite que presas de diferentes tamanhos, estágios e hábitos sejam exploradas como recurso alimentar disponível a algum dos predadores (Miliczky et al. 2000). Semelhante ao encontrado por Rinaldi \& Ruiz (2002), em pomares de seringueira, o grupo das caçadoras errantes foi o mais abundante em ambos os trabalhos, sendo que, no atual estudo, esse grupo esteve representado pelo maior número de indivíduos em $73 \%$ das ocasiões amostrais.

A menor abundância das aranhas construtoras de teia poderia ser atribuída a fatores como grau de especialização alimentar, estrutura do hábitat e perturbação do meio. Conforme Young \& Edwards (1990), esses aspectos são suficientes para ocasionar um número relativamente menor de indivíduos desse grupo em um ambiente. De modo geral, pomares orgânicos facilitam a presença e abundância das aranhas, em especial das caçadoras errantes, as quais, conforme Amalin \& Peña (1999), são mais suscetíveis aos compostos químicos aplicados, por percorrerem a copa ativamente, estando assim mais vulneráveis; contrariamente ao afirmado por Samu et al. (1992), de que as construtoras, por apresentarem o hábito de eventualmente ingerir partes da teia, seriam as mais atingidas.

A presença de Anyphaenidae em plantas de citros é também mencionada por Ott (2003) em levantamento da araneofauna nos vales dos rios Caí e Taquari (RS), onde representou $23,5 \%$ dos indivíduos coletados, valor este inferior ao do presente estudo. Além dos citros, outras culturas de diferentes portes abrigam espécies de Anyphaenidae, sugerindo a plasticidade das espécies dessa família na ocupação de diversos sistemas agrícolas. No Rio Grande do Sul, aranhas anifenídeas têm sido registradas em lavouras de soja e arroz (Corseuil et al. 1994a, b), no estado de São Paulo, em cultivar de seringueira (Rinaldi \& Ruiz 2002) e cana-de-açúcar (Rinaldi et al. 2002).
Considerando apenas os indivíduos adultos, alguns grupos de aranhas destacaram-se, como Theridiidae, pela elevada abundância e riqueza de espécies (20 espécies, Tabela 2). Esse aspecto também foi constatado em pomares de citros na Flórida e no Texas, onde a família foi representada por 13 e seis espécies, respectivamente por Muma (1975) e Brenne et al. (1993). A presença de Theridiidae no citros pode estar ligada à existência de ácaros fitófagos, tripes e afídeos, os quais, segundo Amalin \& Peña (1999), servem como presas para suas espécies. No entanto, seu hábitat não se restringe aos ambientes agrícolas, sendo o mesmo grupo bastante freqüente também em florestas, como evidencia o estudo de Benati et al. (2005) em Mata Atlântica, localizada no município de Salvador, BA. No referido estudo foram coletadas seis espécies de Theridiidae utilizando-se guardachuva entomológico e coleta manual.

Analisando-se os resultados em nível de espécie, considerando apenas os ácaros, Leptus sp. 1 (Erythraeidae) (59,4\%) e Amblyseius saopaulus Denmark \& Muma (Phytoseiidae) (30\%) foram os mais abundantes. Leptus Latreille é um gênero pouco estudado quanto a seu hábito. Espécies de Amblyseius são reconhecidas pelo hábito generalista no consumo de ácaros em pomares de citros da Flórida, incluindo em sua preferência alimentar eriofíideos, tenuipalpídeos e tetraniquídeos (Muma 1971).

Em relação às aranhas, Sphecozone sp. (Linyphiidae), com 21,8\%, e Chrysso pulcherrima (Mello-Leitão) (9,9\%) (Theridiidae) destacaram-se, sendo a primeira, além de abundante, também constante ao longo das amostragens. Schmidt \& Tscharntke (2005) vinculam a maior abundância de Linyphiidae ao início da elevação das temperaturas; entretanto, no presente estudo, a maior abundância de Sphecozone sp. foi registrada em agosto, outubro e novembro (Tabela 2). Estes resultados corroboram o verificado por Ott (2003), utilizando o guarda-chuva entomológico, em pomares de laranja doce, nessa mesma região, onde Sphecozone sp. $(62,94 \%)$ e $C$. pulcherrima $(5,78 \%)$ foram as espécies com maior número de indivíduos. Apesar de Linyphiidae ser comumente ligada a ambientes de solo (Riechert \& Lockley 1984), os registros demonstraram também adaptabilidade a estratos arbóreos.

Quanto à abundância de Acari, o maior valor foi registrado durante o inverno (268) diferindo significativamente apenas do verão $(\mathrm{P}=0,006)$, onde os indivíduos tornaramse praticamente ausentes (cinco). Estudos realizados, especialmente com Phytoseiidae, em relação à época de maior incidência de ácaros predadores, indicam que a variação na abundância de indivíduos pode estar ligada a fatores como precipitação e temperatura. Sato et al. (1994) em citros, em São Paulo, evidenciaram um pico de incidência em junho, quando a temperatura foi inferior à do restante do período (média de $20^{\circ} \mathrm{C}$ ) e a precipitação menor que 25 mm. Ferla \& Moraes (1998) verificaram maior abundância de fitoseídeos em macieira, no Rio Grande do Sul, entre final de dezembro e início de fevereiro, quando ocorreu menor precipitação e as temperaturas foram mais elevadas, em relação ao restante do período amostral. Reis et al. (2000) constataram que em pomares cítricos em Minas Gerais, a predominância de fitoseídeos ocorreu nos meses em que as menores precipitações e temperaturas (abril e setembro) 
foram registradas. Conforme Parra et al. (2003), os ácaros predadores de maneira geral estão presentes no citros em São Paulo, nos meses de janeiro e fevereiro, e de junho a setembro.

A abundância dos ácaros predadores em determinadas estações tem sido atribuída às condições de temperatura, da competição por alimento e da predação de intraguilda (Bostanian et al. 2006). Conforme os autores, as baixas temperaturas favorecem a ocorrência de alguns grupos de ácaros predadores resistentes a tais condições, os quais são capazes de buscar refúgios na planta, aspecto este relatado para algumas espécies de Phytoseiidae.

Em relação ao número total de aranhas (jovens e adultas), a maior abundância foi registrada no outono (748) (Tabela 1), sem apresentar diferença significativa com as demais estações $(\mathrm{P}=0,13)$ e constituindo-se quase que exclusivamente por indivíduos imaturos $(92,6 \%)$. A predominância de jovens em relação aos adultos é um padrão freqüentemente encontrado em levantamentos de araneofauna, corroborrando os estudos de Rinaldi et al. (2002) e Ott (2003).

A alta incidência de aranhas durante o outono provavelmente decorre da disponibilidade dos grupos de presas nessa época, como, por exemplo, de P. citrella. Jesus (2005) estudando a dinâmica populacional desse lepidóptero, de julho de 2001 a junho de 2003, no mesmo pomar onde se desenvolveu o presente estudo, constatou maior densidade de larvas, em ambos os anos, no outono. Esse fato indica maior disponibilidade de presas que poderiam estar favorecendo a manutenção da comunidade de aranhas, visto que algumas espécies, Hibana velox (Becker) (Anyphaenidae), Thachelas volutus (Gertsch) (Corrinidae) e Cheiracanthium inclusum Hentz (Miturgidae), são sabidamente predadoras de $P$. citrella (Amalin et al. 2001).

$\mathrm{Na}$ análise das curvas de rarefação de Araneae (Fig. 3), a maior abundância de indivíduos adultos foi registrada no inverno (119) e primavera (118): para uma subamostra de 51 indivíduos, a primavera também foi a estação com maior riqueza estimada de espécies $(24,81 \pm 2,24)$, diferindo significativamente das demais. O número estimado de

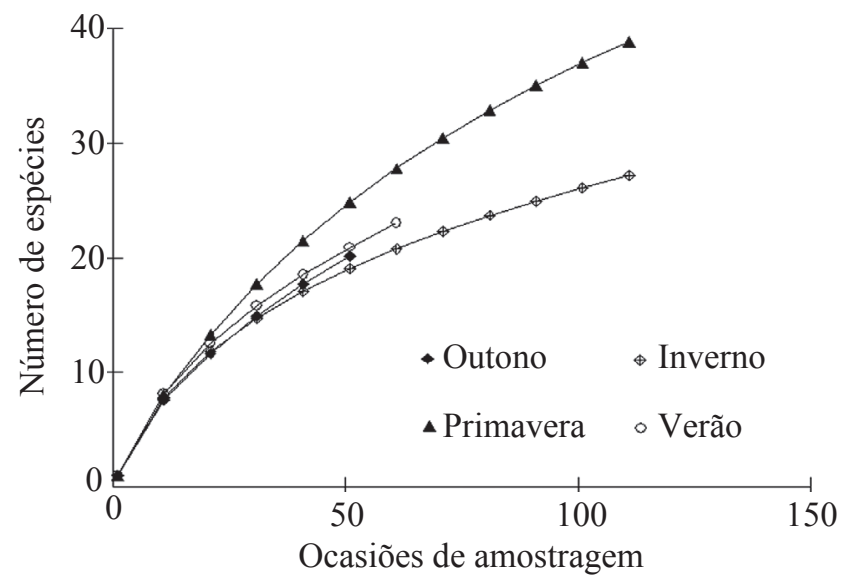

Fig. 3. Curvas de rarefação de aranhas coletadas em pomar orgânico de C. deliciosa var. Montenegrina (29 37'51'’S e $\left.51^{\circ} 28^{\prime} 10^{\prime \prime} \mathrm{W}\right)$, Montenegro, RS, de março de 2004 a março de 2005. espécies no outono $(20,11 \pm 0,80)$, inverno $(19,06 \pm 1,88)$ e verão $(20,91 \pm 1,17)$ não diferiram significativamente entre si.

Das espécies com menor freqüência, 32\% foram representadas por um único indivíduo e $16 \%$ por apenas dois indivíduos durante todo período amostral. Essa proporção é similar à encontrada em outros estudos realizados com aranhas, como o de Flórez (1999), o qual verificou que 50\% das espécies registradas foram representadas por somente um indivíduo. O mesmo autor infere ser esta uma característica da artropodofauna tropical, a existência de um elevado número de espécies com baixa densidade populacional. O padrão de muitas espécies raras é comum em várias comunidades (Silveira Neto et al. 1976). Entretanto, Novotný \& Basset (2000) referem que a ocorrência de espécimes únicos pode estar relacionada a fatores como o método, a época ou número de amostragens insuficientes e ainda à coleta em plantas não preferenciais para a espécie, o que pode proporcionar uma informação imprecisa quanto à sua raridade.

A reduzida abundância no verão, tanto de aranhas quanto de ácaros, pode ter sido decorrente da estiagem ocorrida de dezembro a fevereiro que, de acordo com Berlato (2005), foi a mais intensa ocorrida no Rio Grande do Sul nos últimos 50 anos, e durante a qual choveu cerca de $20 \%$ do que a média histórica vem registrando. Em termos gerais, Acari e Araneae comportaram-se de forma distinta neste estudo quanto à distribuição das espécies, estando os ácaros representados por poucas espécies dominantes e aranhas distribuídas de maneira mais uniforme em relação ao número de indivíduos por espécie (Tabela 2). Os resultados mostram maior abundância de ácaros e aranhas nas estações de temperaturas mais amenas, por outro lado, a baixa precipitação pareceu influenciar negativamente as populações de ambos os grupos.

A comunidade de ácaros predadores diferiu daquelas dos demais estudos quanto à abundância de indivíduos por família, nos quais Phytoseiidae tem sido a mais numerosa (Ferla \& Moraes 1998, Barbosa et al. 2003, Mineiro et al. 2004, Daud \& Feres 2005). No entanto, em termos de riqueza de espécies apresenta-se de maneira semelhante.

Considerando o grupo Araneae, os resultados encontrados no presente estudo seguem o padrão observado em outros trabalhos de levantamento de aranhas da região Neotropical (Rinaldi \& Ruiz 2002, Rinaldi et al. 2002), confirmando Anyphaenidae, Araneidae, Salticidae e Theridiidae como as famílias mais abundantes. Da mesma forma a riqueza de espécies registrada corrobora o referido por Brescovit $e t$ al. (2004) de que Theridiidae, Araneidae e Salticidae são as famílias mais ricas, demonstrando a existência de um mesmo padrão de diversidade de Araneae em agroecossistema.

\section{Agradecimentos}

Ao Prof. Dr. Noeli Juarez Ferla, da Unidade Integrada Vale do Taquari de Ensino Superior (UNIVATES), pela identificação das espécies de Acari. Ao Conselho Nacional de Desenvolvimento Científico e Tecnológico (CNPq), pela concessão da bolsa de estudos ao primeiro e último autores. 


\section{Referências}

Amalin, D.M. \& J.E. Peña. 1999. Predatory spiders in lime orchards and importance in the control of citrus leafminer, Phyllocnistis citrella (Lepidoptera: Gracillariidae). Proc. Fla. Stat. Hort. Soc. 112: 222-224.

Amalin, D.M., J. Reinskind, J.E. Peña \& R. McSorley. 2001. Predatory behavior of three species of sac spiders attacking citrus leafminer. J. Arachnol. 29: 72-81.

Baker, A.S. \& P.A. Selden. 1997. New morphological and host data for the ectoparasitic larva of Leptus hidakai Kawashima (Acari, Acariformes, Erythraeidae). Systematic Parasitol. 36: 183-191.

Barbosa, D.G.F., M.G.C. Goldin Jr., R. Barros \& J.V. Oliveira. 2003. Diversidade de ácaros em aceroleira (Malpighia emarginata A. D. M.) na Universidade Federal Rural de Pernambuco. Neotrop. Entomol. 32: 577-583.

Benati, K.R., J.P. Souza-Alvez, E.A. Silva, M.C.L. Peres \& E.O. Coutinho. 2005. Aspectos comparativos das comunidades de aranhas (Araneae) em dois remanescentes de Mata Atlântica do estado da Bahia, Brasil. Disponível em: http://www. biotaneotropica.org.br/v5n1a/pt/abstract?inventory+BN0060 51a2005. Acesso em $01 \mathrm{dez} .2005$

Berlato, M.A. 2005. Prognósticos e recomendações para o período de julho/agosto/setembro 2005. Disponível em: http://www. emater.tche.br/site/destaques/ptbr/pdf/forum_tempoclima/ reuniaocopaaergs7-5-07-2005.pdf. Acesso em 07 dez. 2005.

Bostanian, N.J., J.M. Hardman, G. Racete, J. Franklin \& J. Lasnier. 2006. Inventory of predacious mites in Quebec commercial apple orchards where integrated pest management programs are implemented. Ann. Entomol. Soc. Am. 99: 536-534.

Breene, R.G., D.A. Dean \& R.L. Meacher Jr. 1993. Spiders and ants of Texas citrus groves. Fla. Entomol. 76: 168-170.

Brescovit, A.D., R. Bertani, R. Pinto Da Rocha \& C.A. Rheims. 2004. Aracnídeos da Estação Ecológica Juréia-Itatins: Inventário preliminar e história natural. p. 198-221. In O.A.V. Marques e W. Duleba. (orgs.), Estação Ecológica Juréia-Itatins: Ambiente físico, flora e fauna. 1 ed. Ribeirão Preto, Holos, 384p.

Colwell, R. K. 2005. EstimateS: Statistic estimation of species richness and shared species from samples. Version 7.5. Disponível em: http://viceroy.eeb.uconn.edu/estimates. Acesso em 05 nov. 2005.

Corseuil, E., A.D. Brescovit \& M.A. Heineck. 1994a. Aranhas associadas à cultura da soja em Eldorado do Sul, Rio Grande do Sul. Biociências 2: 95-105.

Corseuil, E., M.C. De Paula \& A.D. Brescovit. 1994b. Aranhas associadas a uma lavoura de arroz irrigado no município de Itaquí, Rio Grande do Sul. Biociências 2: 49-56.

Daud, R.D. \& R.J.F. Feres. 2005. Diversidade e flutuação populacional de ácaros (Acari) em Mabea fistulifera Mart. (Euphorbiaceae) de dois fragmentos de mata estacional semidecídua em São José do Rio Preto, SP. Neotrop. Entomol. 34: 191-201.
Ferla, J.N. \& G.J. Moraes. 1998. Ácaros predadores em pomares de maçã no Rio Grande do Sul. An. Soc. Entomol. Bras. 27: 649-654.

Ferla, J.N. \& G.J. Moraes. 2002. Ácaros predadores (Acari) em plantas nativas e cultivadas do estado do Rio Grande do Sul, Brasil. Rev. Bras. Zool. 19: 1011-1031.

Flechtmann, C.H.W. 1989. Ácaros de importancia agrícola. $7^{\circ}$ ed. São Paulo, Nobel.189p.

Flórez, E. 1999. Estructura y conposición de un comunidad de arañas (Araneae) en un bosque muy seco tropical de Colombia. Bol. Entomol. Venez. 14: 37-51.

Gravena, S. 1994. Minadora das folhas dos citros: a mais nova ameaça da citricultura brasileira. Laranja 15:397-404.

Green, J. 1996. Spiders in biological control - An Australian perspective. Rev. Suisse Zool. 245-253.

Hajaj, J., D.W. Ross \& A.R. Moldenke. 2000. Importance of habitat structure to arthropod food-wed in Dolglas-fir canopies. Oikos 90: 139-152.

Hammer, O., D.A.T. Harper \& P.D. Ryan. 2001. Past: Palaeonthological Statistics software package for education and data analysis. Palaentologia Electronica 4(1):1-9. version. 1.37. Disponível em: http://palaeo-electronica.org/2001_1/ past/issue1_01.htm

IBGE. Instituto Brasileiro de Geografia Estatística. 2005. Produção agrícola municipal: Culturas temporárias e permanentes. Disponível em: <www.sidra.ibge.gov.br>. Acesso em 10 jan. 2006.

IPAGRO - Instituto de Pesquisas Agronômicas. Secão de Ecologia Agrícola. 1989. Atlas agroclimático do estado do Rio Grande do Sul. Porto Alegre, Ipagro, 102p.

Jesus, C.R. 2005. Dinâmica populacional de Phyllocnistis citrella Stainton, 1856 (Lepidoptera: Gracillariidae) em pomares de duas variedades de citros em Montenegro, RS. Tese de doutorado, Faculdade de Agronomia, Universidade Federal do Rio Grande do Sul, 102p.

McAleece, N. 1997. Biodiversity Professional 2.0. The Natural History Museum and the Scotish Association for Marine Science. Disponível em: <http://www.nhm.ac.uk/zoology/ bdpro>. Acesso em 12 dez. 2003.

McMurtry, J.A. \& B.A. Croft. 1997. Life-styles of Phytoseiid mites and their roles in biological control. Ann. Rev. Entomol. 42: 291-321.

Merfield, C.N., S.D. Wratten \& S. Navntoft. 2004. Video analysis of predation by polyphagous invertebrate predators in the laboratory and field. Biol. Control 29: 5-13.

Miliczky, E.R., C.O. Calkins \& D.R. Horton. 2000. Spider abundance and diversity in apple orchards under three insect pest management programmes in Washington State, U. S. A. Agric. Forest Entomol. 2: 203-215.

Mineiro, J.L., A. Raga, \& A.C. Lofego. 2004. Ocorrência de ácaros (Arachnida: Acari) em aceroleira (Malpighia emarginata A. DC.) no estado de São Paulo. Arq. Inst. Biol. 7: 271-274. 
Moraes, G.J. 1992. Perspectivas para o uso de predadores no controle de ácaros fitófagos no Brasil. Pesq. Agropec. Bras. 27: $263-270$.

Moraes, G.J. 2002. Controle biológico de ácaros fitófagos com ácaros predadores, p. 225-237. In J.R.P. Parra, S.M. Botelho, B.S. Corrêa-Ferreira \& J.M.S. Bento (eds.), Controle biológico no Brasil: Parasitóides e predadores. São Paulo, Manole, $626 \mathrm{p}$.

Moreno, C.E. 2001. Métodos para medir la biodiversidad. M \& T- Manuales y Tesis SEA. Zaragoza, Cited/ Unesco \& SEA. v. 1,84 p.

Muma, M.H. 1971. Food habits of Phytoseiidae (Acari: Mesostigmata) including common species on Florida citrus. Fla. Entomol. 54: 21-34.

Muma, M.H. 1975. Spiders in Florida citrus groves. Fla. Entomol. 58: $82-90$

Novotný, V. \& Y. Basset. 2000. Rare species in communities of tropical insect herbivores: Pondering the mystery of singletons. Oikos 89: 564-572.

Ott, A.P. 2003. Levantamento de cigarrinhas e aranhas em pomares de laranja Valência nos vales do Caí e Taquari, RS, Brasil. Tese de Doutorado, Faculdade de Agronomia, Universidade Federal do Rio Grande do Sul, 76p.

Parra, J.R.P., H.N. Oliveira, A.S. Pinto. 2003. Guia ilustrado de pragas e insetos benéficos dos citros. Piracicaba, A. S. Pinto, $140 \mathrm{p}$.

Raga, A., M.E. Sato, L.C. Cerávolo \& A.C. Rossi. 1996. Distribuição de ácaros predadores (Phytoseiidae) em laranjeira (Citrus sinensis L. Osbeck). Rev. Ecossistema 21: 23-25.

Riechert, S.E. \& T. Lockley. 1984. Spiders as biological control agents. An. Rev. Entomol. 29: 299-320.

Reis, P.R., L.G. Chiavegato, E.B. Alves \& E.O. Sousa. 2000. Ácaros da família Phytoseiidae associados aos citros no município de Lavras, Sul de Minas Gerais. An. Soc. Entomol. Bras. 29: 95-104.
Rinaldi, I.M.P., B.P. Mendes \& A.B. Candy. 2002. Distribution and importance of spiders inhabiting a Brazilian sugar cane plantation. Rev. Bras. Zool. 19: 271-279.

Rinaldi, I.M.P. \& G.R.S. Ruiz. 2002. Comunidades de aranhas (Araneae) em cultivos de seringueira (Hevea brasiliensis Muell. Arg) no Estado de São Paulo. Rev. Bras. Zool. 19: 781-788.

Samu, F., A.M. Graham, D. Lake \& F. Vollrath. 1992. Spider webs are efficient collectors of agrochemical spray. Pestic. Sci. 36: 47-51.

Sato, M.E., A. Raga, L.C. Cerávolo, A.C. Rossi \& M.R. Potenza. 1994. Ácaros predadores em pomar cítrico de Presidente Prudente, estado de São Paulo. An. Soc. Entomol. Brasil 23: 435-441.

Schmidt, M.H. \& T. Tscharntke. 2005. Landscape context of sheetweb spider (Araneae: Linyphiidae) abundance in cereal fields. J. Biogeogr. 32: 467-473.

Silveira Neto, S., O. Nakano, D. Barbin \& N.A. Villa Nova. 1976. Manual de ecologia dos insetos. São Paulo, Agronômica Ceres, 419p.

Smith, I.M., E.E. Lindquist \& V. Behan-Pelletier. 1998. Mites (Acari). In I.M. Smith \& G.G. Scudder (eds.), Assessment of species diversity in the Montane Cordillera Ecozone. Burlington: Ecological Monitoring and Assessment Network, 1998. Disponível em: http://www.naturewatch.ca/eman/ reports/publications/99_montane/mites/mites05.html. Acesso em 01 fev. 2007.

Uetz, G.W., J. Halaj \& A.B. Cady. 1999. Guild structure of spiders in major crops. J. Arachnol. 27: 270-280.

Young, O.P. \& G.B. Edwards. 1990. Spider in United States Field crops and their potential effect on crop pests. J. Arachnol. 18: 1-127.

Received 13/X/06. Accepted 05/V/07. 